\title{
Attenuating Immune Response of Macrophage by Enhancing Hydrophilicity of Ti Surface
}

\author{
Xiaohan Dai, ${ }^{1,2}$ Yan Wei, ${ }^{2}$ Xuehui Zhang, ${ }^{3}$ Song Meng, ${ }^{2}$ Xiaoju Mo, ${ }^{2}$ Xing Liu, ${ }^{4}$ \\ Xuliang Deng, ${ }^{2}$ Li Zhang, ${ }^{5}$ and Xuming Deng ${ }^{1}$ \\ ${ }^{1}$ Key Laboratory of Zoonosis, Ministry of Education, Institute of Zoonosis, College of Veterinary Medicine, Jilin University, \\ Changchun 130062, China \\ ${ }^{2}$ Department of Geriatric Dentistry, Peking University School and Hospital of Stomatology, Beijing 100081, China \\ ${ }^{3}$ Department of Dental Materials, Peking University School and Hospital of Stomatology, Beijing 100081, China \\ ${ }^{4}$ First Clinical Division, Peking University School and Hospital of Stomatology, Beijing 100034, China \\ ${ }^{5}$ Research Center for Nano-Biomaterials, Analytical \& Testing Center, Sichuan University, Chengdu 610064, China
}

Correspondence should be addressed to Li Zhang; zhangli9111@126.com and Xuming Deng; dengxm@jlu.edu.cn

Received 25 October 2014; Revised 4 January 2015; Accepted 15 January 2015

Academic Editor: Dawei Liu

Copyright (C) 2015 Xiaohan Dai et al. This is an open access article distributed under the Creative Commons Attribution License, which permits unrestricted use, distribution, and reproduction in any medium, provided the original work is properly cited.

Immune responses can determine the in vivo fate of implanted materials. The strategy for developing implants has shifted towards using materials with immunomodulatory activity. However, the immunoregulatory effect of hydrophilicity of titanium surface on the macrophage behavior and its underlying mechanism remain poorly understood. Here, the Ti surface hydrophilicity-dependent behavior of murine RAW264.7 macrophages was investigated in vitro. Two laboratory models with significantly different surface hydrophilicity and similar roughness were established with Ti-polished and $\mathrm{Ti}-\mathrm{H}_{2} \mathrm{O}_{2}$ surfaces. The results of cell morphology observation showed that the Ti- $\mathrm{H}_{2} \mathrm{O}_{2}$ surface yielded enhanced cell adhesion and less multinucleated cell formation. CCK-8 assay indicated that the growth rate of macrophage on Ti- $\mathrm{H}_{2} \mathrm{O}_{2}$ surface is higher than that of Ti-polished. ELISA assay result revealed lower level of proinflammatory factor TNF- $\alpha$ and higher level of anti-inflammatory factor IL-10 on the Ti- $\mathrm{H}_{2} \mathrm{O}_{2}$ surface compared to Ti-polished. Subsequently, immunofluorescence and western blotting analysis showed that activation of the NF- $\kappa$ B-TNF- $\alpha$ pathway might be involved in the modulation of the immune response by surface hydrophilicity. Together, these results suggested that relative high hydrophilic Ti surface might attenuate the immune response of macrophage by activating NF- $\kappa \mathrm{B}$ signaling. These findings could provide new insights into designing implant devices for orthopedic applications.

\section{Introduction}

Because of excellent mechanical and corrosion resistance properties, titanium- (Ti-) based materials have been widely used in making dental and orthopedic implants $[1,2]$. However, immune responses mediated by inflammatory cells have been found to significantly impact the biocompatibility and function of implanted medical devices or prostheses and can ultimately precipitate their clinical failure $[3,4]$. The paradigm for developing an ideal implant material has been shifted using from inert to immunomodulatory materials, emphasizing the importance of immune cells in the evaluation of a candidate material [5]. Macrophages, the major effector cells in the immune response to implants, have been reported to determine the long-term immune response and outcome of an inflammatory reaction $[6,7]$. Modulation of adhesion and activation of macrophages with anti-inflammatory drugs can reduce immune responses [8]. However, in the clinic the long-term systematic use of drugs can compromise host defenses, leading to impaired immune responses and an increased potential for infection [9]. To avoid this problem, appropriate surface modifications of implants have been considered as an alternative approach to inhibit or attenuate inflammation reaction temporarily for achieving high success rates [10-12].

Recently, several types of surface modification methods have been introduced to slow macrophage-mediated immune 
responses triggered by Ti-based implants, including coatings, sand blasting, and acid etching [13-15]. However, delamination of the coating was found to sometimes occur during implant placement or in load bearing situations, which was probably caused by poor stability of the coating bound to the substrate [13]. Additionally, normal bone mineralization can be inhibited by the release of constituents, such as $\mathrm{Al}_{2} \mathrm{O}_{3}$ or $\mathrm{Ta}_{2} \mathrm{O}_{5}$, from the material when they were modified by sand blasting [14]. The etched implant surface has been shown to be capable of promoting osseointegration because of hydrophilicity [15]. Furthermore, surface hydrophilicity of Ti-based implants has been established to modulate osteogenic cell adhesion, proliferation, and differentiation [16]. Therefore, it can be hypothesized that incorporating surface hydrophilicity might be a promising alternative for regulating macrophage behavior. Nevertheless, little attention has been paid to the immunoregulatory effect of surface hydrophilicity of titanium on the macrophage behavior and data about this topic has been needed.

Herein, the purpose of this work is to investigate the effects of the hydrophilicity of $\mathrm{Ti}$ surface on macrophage behavior in vitro. Two kinds of Ti surfaces with different hydrophilicity including polished $\mathrm{Ti}$ surface (Ti-polished) and etched Ti surface $\left(\mathrm{Ti}-\mathrm{H}_{2} \mathrm{O}_{2}\right)$ were used in present study. The morphology, roughness, and wettability of the Ti surfaces were firstly characterized. The morphology of macrophage RAW264.7 cells on the Ti surfaces was observed by scanning electron microscopy (SEM) and confocal laser scanning microscopy (CLSM). Cell proliferation was assessed by CCK8 assay. The activation status of macrophages was quantified by measuring TNF- $\alpha$ and IL-10 secretion by ELISA assay. Subsequently, the underlying mechanism of modulation of surface hydrophilicity on the immune response was explored by both immunofluorescence and western blotting analysis.

\section{Materials and Methods}

2.1. Samples Preparation. Ti disks with $20 \mathrm{~mm}$ in diameter and $2 \mathrm{~mm}$ thick were used to prepare two kinds of different $\mathrm{Ti}$ surfaces including hydrophilic $\left(\mathrm{Ti}-\mathrm{H}_{2} \mathrm{O}_{2}\right)$ and polished $(\mathrm{Ti}-$ polished). Ti-polished surfaces were prepared by polishing with sand paper from 400 to 1500 grit. $\mathrm{Ti}-\mathrm{H}_{2} \mathrm{O}_{2}$ surfaces were obtained via immersing the Ti disks in $10 \% \mathrm{H}_{2} \mathrm{O}_{2}$ solution for $24 \mathrm{~h}$.

2.2. Sample Characterization. The surfaces were imaged by scanning electron microscope (SEM, Hitachi S-4800, Tokyo, Japan) at a $15 \mathrm{kV}$ accelerating voltage. Atomic force microscopy (AFM, SPA-300HV, Seiko, Tokyo, Japan) was performed to measure the surface roughness parameter $\mathrm{Ra}$ over a $10 \times 10 \mu \mathrm{m}$ area. X-ray photoelectron spectroscopy (XPS, Thermo V6 Escalab 250, West Sussex, UK) was carried out to analyze element composition of these $\mathrm{Ti}$ surfaces. Surface water contact angles of Ti materials were measured using a drop shape analysis system (JY-82A, China).

2.3. Cell Culture. Murine macrophage-like RAW264.7 cells were obtained from Cell Resource Center, IBMS,
CAMS/PUMC, and cultured in DMEM medium with $10 \%$ FBS (Gbico, Carlsbad, CA, USA). After two passages, cells were plated at $2.0 \times 10^{5}$ cells per well in 24 -well plates.

\subsection{Cell Morphology Observation}

2.4.1. Scanning Electron Microscopy (SEM). Cells were incubated on Ti disks for 1,3 , and 7 days. After incubation, cells were fixed with $2.5 \%$ glutaraldehyde and incubated with $0.18 \mathrm{~mol} / \mathrm{L}$ sucrose solution. Samples were dehydrated through a graded ethanol series (30-100\%) and air-dried.

2.4.2. Confocal Laser Scanning Microscopy (CLSM). Cells were cultured on Ti disks for 7 days. Cells were then fixed with $4.0 \%$ paraformaldehyde, treated with Alexa Fluor 546phalloidin $(50 \mu \mathrm{g} / \mathrm{mL})$ for $1 \mathrm{~h}$ at room temperature, and then stained with DAPI for 10 min according to the manufacturer's directions. Images of the stained cells were acquired using CLSM (Zeiss, LSM 780, Oberkochen, Germany).

2.5. Cell Proliferation Assay. To assess macrophage activity on Ti disks, cell counting kit-8 (CCK-8) was performed on days 1, 3, 5, and 7. According to the manufacturer's directions, CCK-8 solution was added to each well of the plate at a ratio of $1: 10$ and then was incubated for $2 \mathrm{~h}$ in an incubator. After incubation, absorbance was measured using a microplate reader (Bio-Rad, Hercules, CA, USA).

2.6. Cell Cytokine Secretion Examination. To analyze cytokine secretion, cells were cocultured with Ti disks and supernatants were collected at days 1,3,5, and 7. The levels of TNF- $\alpha$ and IL-10 in the supernatants were quantified using an ELISA kit (R\&D Systems, Minneapolis, MN, USA) according to the manufacturer's directions.

2.7. Assessment of $N F-\kappa B$. In accordance with the manufacturer's directions, cells grown on Ti disks for 3 days were fixed, blocked, and incubated with anti-NF- $\kappa$ B p 65 primary antibody at $4^{\circ} \mathrm{C}$ overnight. Then samples were treated with an appropriate Cy3-conjugated secondary antibody for $1 \mathrm{~h}$ and stained with DAPI for $5 \mathrm{~min}$ at room temperature. A confocal microscopy (Zeiss, LSM 780, Oberkochen, Germany) was used to observe NF- $\kappa \mathrm{B}$ activation.

2.8. Statistical Analysis. Data were analyzed using an independent Student's $t$-test and are presented as means \pm standard deviation (S.D.). $P<0.05$ was considered to indicate a statistically significant difference.

\section{Results and Discussion}

Given the importance of macrophages in bone dynamics, some studies have investigated interactions between bone substitute biomaterials (e.g., bioceramics, polymers, or titanium) and macrophages [11, 17, 18]. However, in the field of implant materials research, the immunoregulatory effect of surface hydrophilicity of titanium on the macrophage 

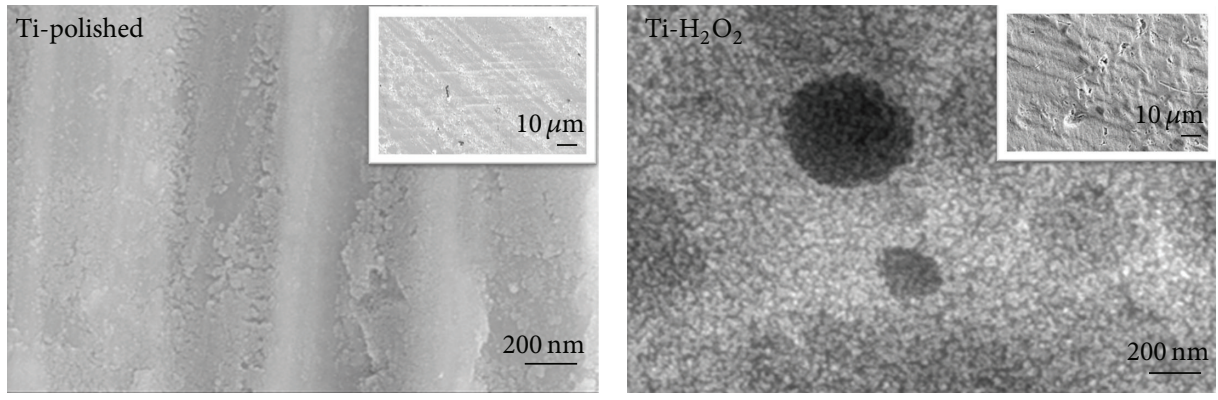

(a)
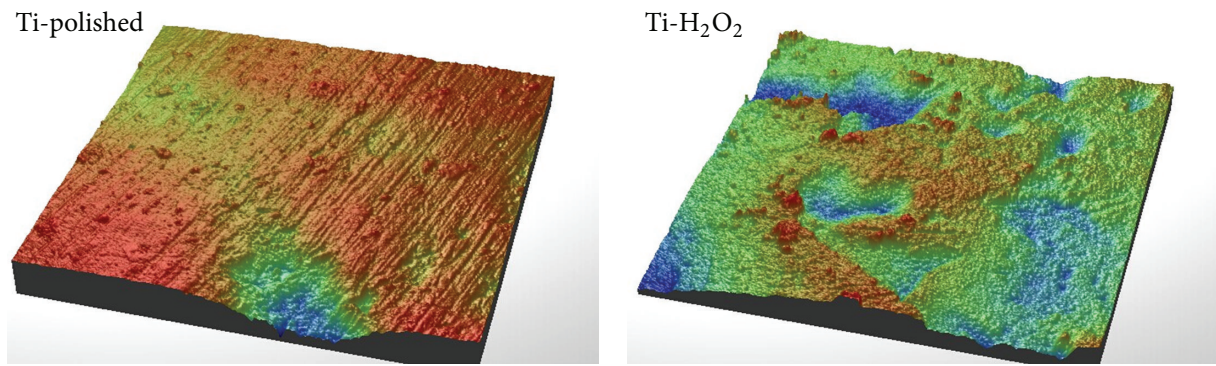

(b)

Figure 1: Scanning electron microscopy (a) and atomic force microscopy (b) images of the Ti-polished and Ti- $\mathrm{H}_{2} \mathrm{O}_{2}$ surfaces.

behavior and its underlying mechanism still remain largely unknown. To devise a strategy for Ti surface design for clinical regenerative therapies, additional data regarding the effects of surface hydrophilicity on immune responses and the underlying mechanisms involved will be required. The results in present study demonstrated that a more hydrophilic $\mathrm{Ti}$ surface might more favorably attenuate macrophage immune responses via NF- $\kappa \mathrm{B}-\mathrm{TNF}-\alpha$ signaling.

The SEM observation clearly indicated that $\mathrm{Ti}-\mathrm{H}_{2} \mathrm{O}_{2}$ possessed a nanostructure with a uniformly distributed nanoparticle compared to Ti-polished surface (Figure 1(a)). As shown in Table 1, AFM images showed that the two kinds of Ti surfaces shared similar roughness that $\mathrm{Ra}$ of Ti-polished and $\mathrm{Ti}-\mathrm{H}_{2} \mathrm{O}_{2}$ were $23.25 \pm 5.89 \mathrm{~nm}$ and $87.13 \pm 23.72 \mathrm{~nm}$. While Ti- $\mathrm{H}_{2} \mathrm{O}_{2}$ surface $\left(35.21 \pm 3.08^{\circ}\right)$ was found to be more hydrophilic than the Ti-polished surface $\left(64.46 \pm 2.384^{\circ}\right)$. The quantified element composition given by XPS indicated a little increment in the O1s peak for the hydrophilic Ti$\mathrm{H}_{2} \mathrm{O}_{2}$ surface compared to hydrophobic Ti-polished surface, supported by atomic ratios of oxygen (Figure 2 and Table 2). The results were consistent with previous report that $\mathrm{O}_{2}{ }^{-}$and $-\mathrm{OH}$ group would be generated on surface when treated with $\mathrm{H}_{2} \mathrm{O}_{2}$ solution [19]. Thus, the two kinds of Ti surface models used in our work presented significantly different surface hydrophilicity and similar roughness.

The proliferation rate of cells on the Ti surfaces partially reflects biocompatibility of the materials, which is essential for implant survival. Mouse macrophage RAW264.7 cells viability on different $\mathrm{Ti}$ surfaces was measured by CCK8 assay for 1, 3, 5, or 7 days (Figure 3). Overall, relative high hydrophilic Ti- $\mathrm{H}_{2} \mathrm{O}_{2}$ surface resulted in enhanced cell proliferation. Although cell viabilities on different surfaces
TABLE 1: Water contact angle and surface roughness of the $\mathrm{Ti}$ samples.

\begin{tabular}{lcc}
\hline Sample & Contact angle $\left(^{\circ}\right)$ & $\mathrm{Ra}(\mathrm{nm})$ \\
\hline $\mathrm{Ti}-\mathrm{H}_{2} \mathrm{O}_{2}$ & $35.21 \pm 3.08$ & $87.13 \pm 23.72$ \\
Ti-polished & $64.46 \pm 2.38$ & $23.25 \pm 5.89$ \\
\hline
\end{tabular}

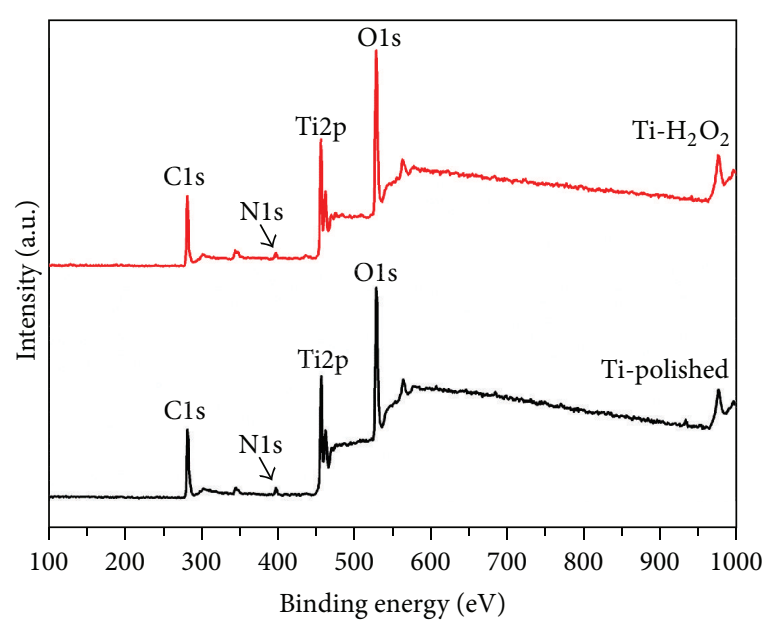

FIGURE 2: X-ray photoelectron spectroscopy analysis of the Tipolished and $\mathrm{Ti}-\mathrm{H}_{2} \mathrm{O}_{2}$ surfaces.

were similar after 1 day of culture, significantly higher cell viabilities were found on hydrophilic Ti- $\mathrm{H}_{2} \mathrm{O}_{2}$ surfaces compared to hydrophobic Ti-polished surfaces after culture for 3 days. Additionally, this trend continued to be maintained 
TABLE 2: Element distribution recorded by XPS analysis.

\begin{tabular}{lcc}
\hline & Ti- $\mathrm{H}_{2} \mathrm{O}_{2}$ & Ti-polished \\
\hline $\mathrm{C} 1 \mathrm{~s}$ & $42.31 \%$ & $44.71 \%$ \\
$\mathrm{Ti} 2 \mathrm{p}$ & $13.35 \%$ & $13.15 \%$ \\
$\mathrm{~N} 1 \mathrm{~s}$ & $2.58 \%$ & $2.72 \%$ \\
O1s & $41.77 \%$ & $39.44 \%$ \\
F1s & 0 & 0 \\
\hline
\end{tabular}

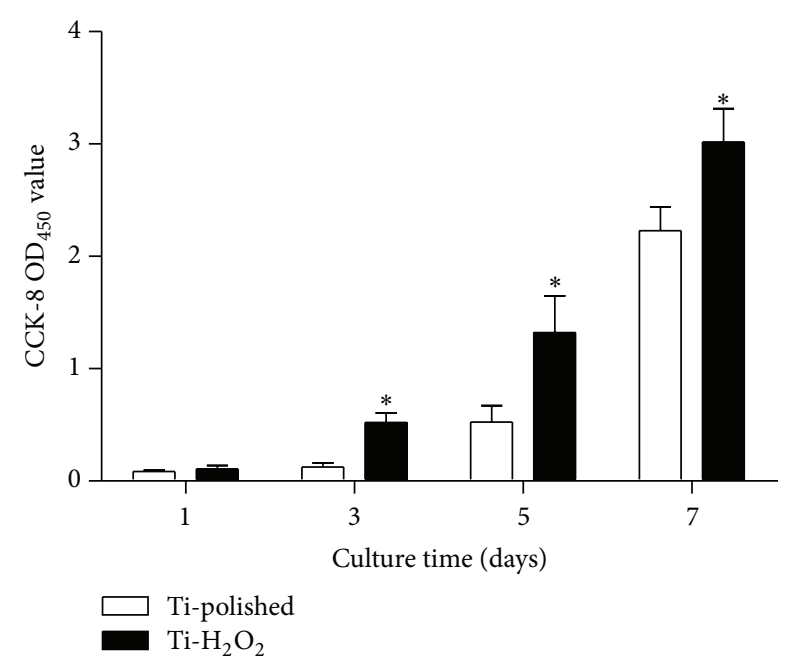

FIgURE 3: The effect of surface hydrophilicity on RAW264.7 cells viability measured by CCK $-8\left({ }^{*} P<0.05\right)$.

at days 5 and 7. These data demonstrate that hydrophilic Ti$\mathrm{H}_{2} \mathrm{O}_{2}$ surfaces are more biocompatible than hydrophobic Tipolished surfaces. The excellent biocompatibility of Ti- $\mathrm{H}_{2} \mathrm{O}_{2}$ surfaces for macrophage may be benefit for wound healing. Previous studies also indicated that improved wound healing was associated with enhanced macrophage recruitment [20].

The influence of materials on macrophage morphology played an important role in the assessment of materials immunoregulatory property. Cell shape changes have been reported to be related to different functional states of cells, such as proliferation, nuclear organization, and differentiation [21-23]. Additionally, cell shape was found to modulate the phenotypic polarization of macrophages. In this study, we clearly observed different initial cell adhesion behaviors of macrophages on two types of Ti surfaces after 1 day of culture (Figure 4(a)). More filopodia were observed on hydrophobic Ti-polished surfaces than on those of hydrophilic Ti- $\mathrm{H}_{2} \mathrm{O}_{2}$ surfaces. Filopodia are specialized macrophage adhesion structures that can form in the early stages of cell adhesion and consist of punctuate F-actin on plasma membrane extensions [24], which facilitate attachment and migration [25, 26]. It has been proposed that a round shape might indicate a resting nonactivated macrophage, whereas the presence of multiple filopodia represents an activated macrophage [26]. Therefore, the difference in filopodia numbers clearly suggested that macrophages would be more prone to become activated on the hydrophobic $\mathrm{Ti}-\mathrm{H}_{2} \mathrm{O}_{2}$ surface than on the hydrophilic Ti-polished.
After 7 days of culture, SEM observation showed that several suspected FBGCs formed by cell fusion appeared on Ti-polished surfaces, whereas only elongated cells with numerous cellular projections were present on $\mathrm{Ti}-\mathrm{H}_{2} \mathrm{O}_{2}$ surfaces (Figure 4(a)). Furthermore, immunofluorescence staining of cytoskeleton organization and CLSM analysis of nuclei confirmed that more FBGCs formed on hydrophobic Ti-polished surfaces than on hydrophilic Ti- $\mathrm{H}_{2} \mathrm{O}_{2}$ surfaces (Figure 4(b)). This phenomenon was consistent with previous work of Collier et al. who reported that hydrophilic surfaces exert a robust inhibitory effect on FBGCs formation [25]. The formation of FBGCs in response to implanted biomaterials or medical devices, also known as a foreign body reaction, has been implicated in the degradation and cracking of implanted material and in initiating the formation of fibrous capsules that surround the implanted biomaterials [27, 28]. FBGCs with multiple nuclei might release profibrotic factors, including transforming growth factor beta (TGF- $\beta$ ) and platelet-derived growth factor (PDGF), which could in turn activate fibroblasts and endothelial cells to facilitate the subsequent fibrotic encapsulation and inflammatory response [21, 29]. Thus, increased FBGCs formation on hydrophobic Tipolished surfaces might imply a more serious inflammatory response of macrophages, and hydrophilic Ti- $\mathrm{H}_{2} \mathrm{O}_{2}$ surfaces might be more beneficial for inhibiting inflammation.

Notably, the two types of $\mathrm{Ti}$ surfaces also yielded macrophages with significantly different cell morphologies. More macrophages with spindles appeared on $\mathrm{Ti}-\mathrm{H}_{2} \mathrm{O}_{2}$ surface, whereas almost all macrophages exhibited a round cell appearance with multiple filopodia on Ti-polished surfaces (Figure 4(b)). These results suggested that Ti- $\mathrm{H}_{2} \mathrm{O}_{2}$ surfaces might promote the polarization of macrophages towards a prohealing macrophage phenotype, implying that they can exert anti-inflammatory activities and improve wound healing responses. Our results were also consistent with previous report that prohealing cells exhibited a more elongated shape compared to proinflammatory cells [30]. By contrast, the round cell morphology on Ti-polished surfaces might be a phenotypic marker of proinflammatory macrophages, leading to fibrotic responses and inflammation around any materials.

It has been well established that the immune system is closely related to skeletal development and that they share many common cytokines [31]. Those cytokines can mediate macrophage-dependent inflammatory cascades that are triggered by innate immune activation and act to influence implant integration and osteogenesis [32-35]. Proinflammatory cytokines can enhance osteoclast differentiation and resorbing activity and inhibit osteoblast activity and bone formation, whereas anti-inflammatory cytokines exert opposite effects [33-35]. TNF- $\alpha$, a key proinflammatory regulator that is mainly released by stimulated macrophages, contributes to fibrosis via TNF receptor 2 , which then acts through extracellular signal-regulated kinase 1/2 (ERK1/2) to stimulate cell proliferation and inhibit collagen degradation [33]. TNF- $\alpha$ was also found to activate the ERK-specific mitogen-activated protein kinase (MAPK) pathway, leading to increased TGF- $\beta 1$ production, which can facilitate fibrosis 

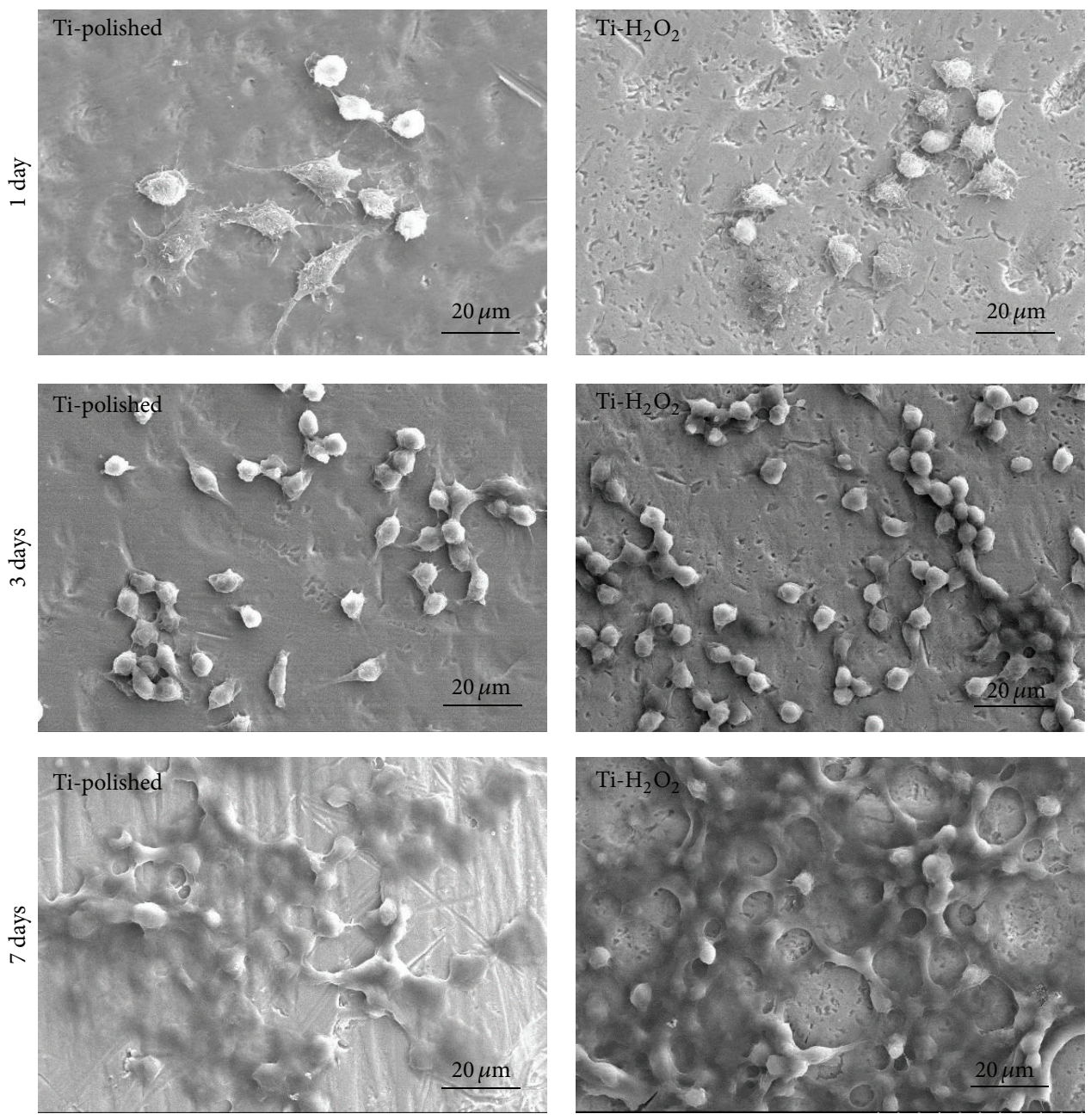

(a)
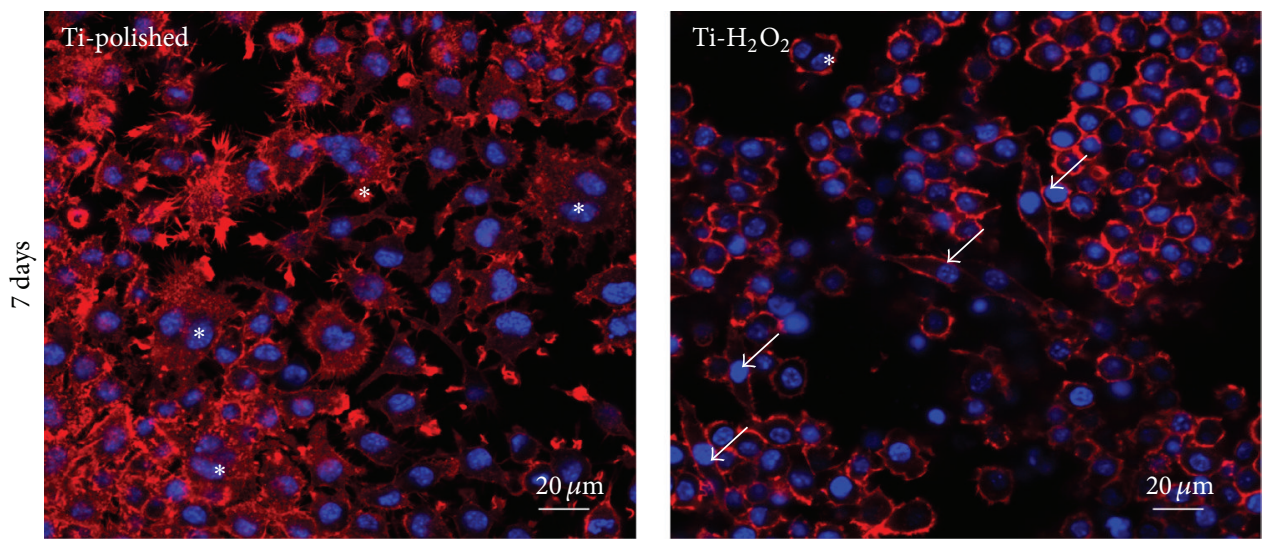

(b)

FIGURE 4: The influence of surface hydrophilicity on cell morphology and FBGCs formation. (a) Scanning electron microscopy images of macrophages cultured on Ti-polished or Ti- $\mathrm{H}_{2} \mathrm{O}_{2}$ surfaces for 1, 3, and 7 days. (b) The cytoskeleton organization of macrophages cultured on Ti-polished and Ti- $\mathrm{H}_{2} \mathrm{O}_{2}$ surfaces for 7 days. White asterisks indicate FBGCs. White arrow indicates the spindle cells. F-actin and cell nuclei were immunostained with Alexa Fluor 546-phalloidin (red) and DAPI (blue), respectively. 


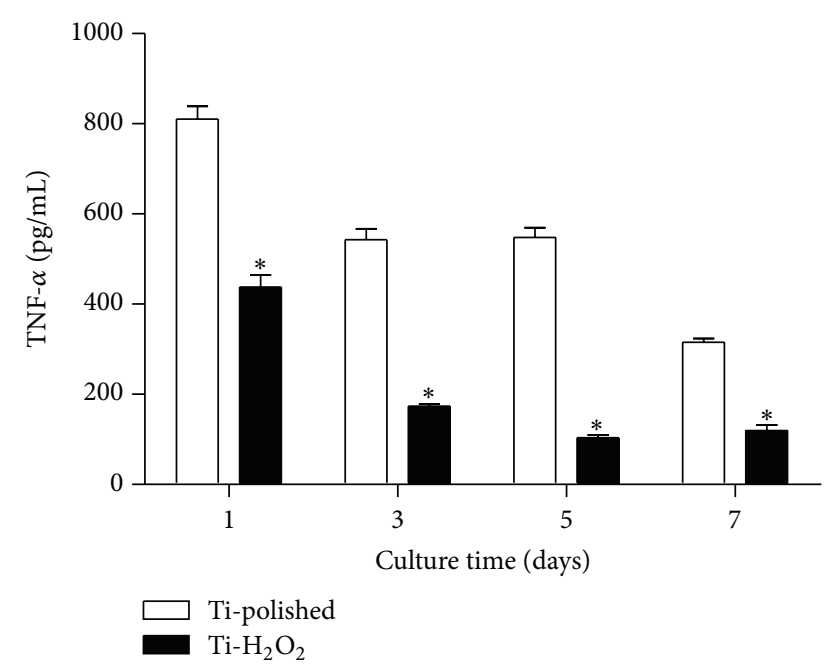

(a)

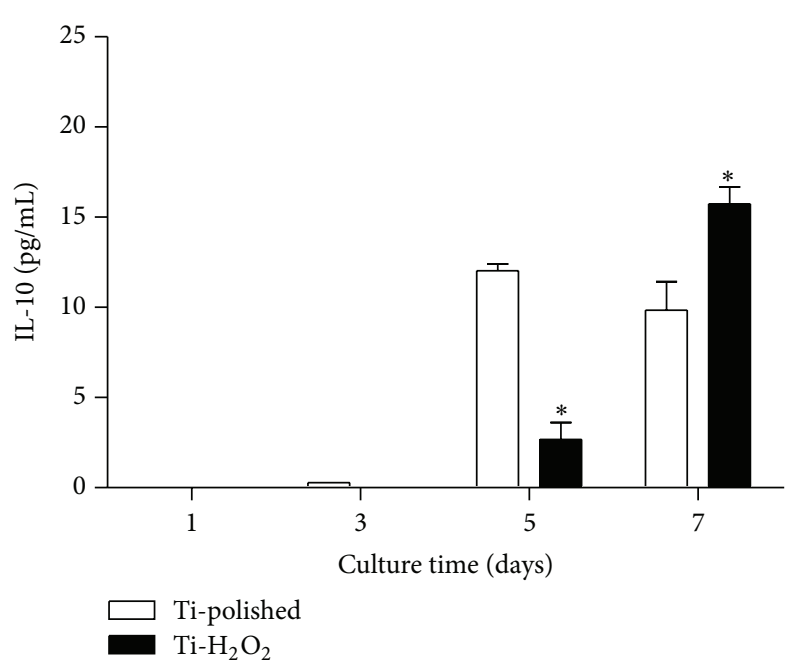

(b)

FIGURE 5: The levels of secreted TNF- $\alpha$ (a) and IL-10 (b) in the supernatants of macrophages cultured on different surfaces for 1, 3, 5, and 7 days $\left({ }^{*} P<0.05\right)$.

[36]. The ELISA quantitative analysis showed that TNF$\alpha$ secretion was significantly increased on hydrophobic Tipolished surfaces compared to hydrophilic Ti- $\mathrm{H}_{2} \mathrm{O}_{2}$ surfaces at all time points (Figure 5(a)). Production of the proinflammatory cytokine TNF- $\alpha$ peaked on both Ti surfaces after 1 day of culture and then decreased with the time, suggesting that a robust inflammatory response occurred at an early stage of culture. IL-10 is a typical anti-inflammatory cytokine that plays a critical role in suppressing proinflammatory cytokines [35]. In contrast to the pattern of proinflammatory cytokine secretion, production of the anti-inflammatory cytokine IL-10 increased in a time-dependent manner on both Ti surfaces (Figure 5(b)). These results indicated that the surface wettability of Ti implants has a profound effect on the immune response of macrophages. Our results are also in line with previous studies. Lee et al. reported that hydrophilic titanium elicited fewer inflammation reactions [37]. Tsang et al. demonstrated that hydrophilic carbon nanofibers (CNFs) caused a weaker macrophage-dependent inflammatory response than hydrophobic CNFs [38]. Taken together, hydrophilic $\mathrm{Ti}-\mathrm{H}_{2} \mathrm{O}_{2}$ surfaces may potentially be better osteogenesis-promoting materials, due to the ability to inhibit the production of proinflammatory cytokines and promote anti-inflammatory molecule secretion.

The confirmation that macrophage behavior can be affected by different hydrophilic $\mathrm{Ti}$ surfaces prompted us to further explore the possible mechanism underlying the hydrophilicity-dependent inflammatory response to implanted materials. Activation of the NF- $\kappa \mathrm{B}$ pathway, a key intracellular regulator of inflammatory signaling, has been known to promote the secretion of proinflammatory cytokines, including TNF- $\alpha$ and IL- $1 \beta[30,31]$. In our work, the significantly different levels of TNF- $\alpha$ production on these two $\mathrm{Ti}$ surfaces implied that the NF- $\kappa \mathrm{B}$ pathway might be critical in the hydrophilicity-dependent immune response. The NF- $\kappa$ B family consists of five members- $p 50$, p52, p65 (RelA), c-Rel, and RelB-that share an N-terminal Rel homology domain (RHD) [32]. Most of these proteins can form different NF- $\kappa$ B homodimers and heterodimers. Among the heterodimers, p50/p65 heterodimers are considered to be key mediators of gene expression [33]. Therefore, p65 was chosen in present research as the mark for NF$\kappa \mathrm{B}$ expression. It can be seen from immunofluorescence images and western blotting results in our work that more NF- $\kappa$ B p 65 was present in macrophages cultured on $\mathrm{Ti}$ polished surfaces than in those cultured on $\mathrm{Ti}-\mathrm{H}_{2} \mathrm{O}_{2}$ surfaces (Figure 6). Combined with inflammatory cytokines secretion results, we can conclude that $\mathrm{NF}-\kappa \mathrm{B}$ signaling may be involved in the regulation effects of hydrophilic Ti surfaces on the acute phase inflammation response of macrophage. Conclusively, these findings could provide an approach to optimize the Ti implant materials for desired bone integration and orthopedic applications.

\section{Conclusions}

In this study, Ti surface hydrophilicity-dependent immune response and underlying mechanism were explored. The results showed that more hydrophilic Ti surface $\left(\mathrm{Ti}-\mathrm{H}_{2} \mathrm{O}_{2}\right)$ yielded enhanced cell adhesion, increased proliferation, and less multinucleated cells formation. The hydrophilic Ti- $\mathrm{H}_{2} \mathrm{O}_{2}$ surfaces that exhibited the inhibition in the production of pro-inflammatory cytokines and the promotion in the secretion of anti-inflammatory molecules may be more beneficial to serve as potentially osteogenesis-promoting materials. Moreover, it was revealed that hydrophilic Ti surface might be more favorable in attenuating macrophage immune response via $\mathrm{NF}-\kappa \mathrm{B}$ signaling. These results may provide new insight in surface-designing of novel implant devices. 

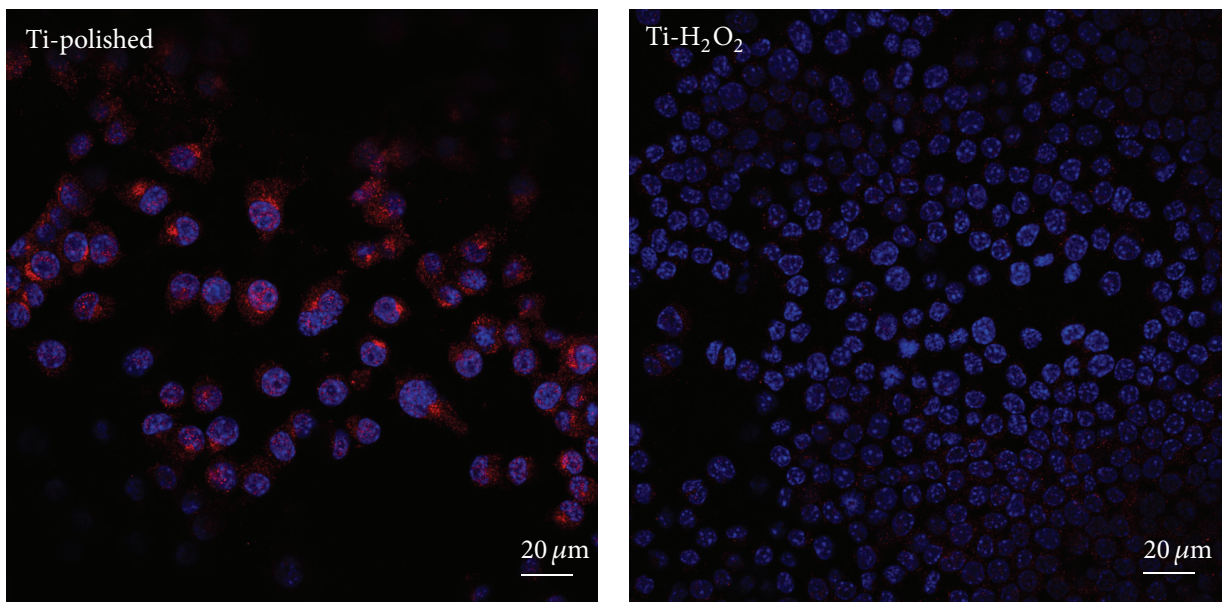

(a)

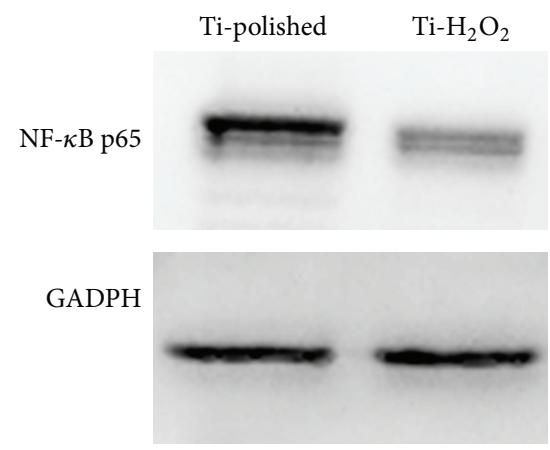

(b)

FIGURE 6: Immunofluorescence images (a) and western blotting images (b) of NF- $\kappa$ B p65 in macrophage nuclei. NF- $\kappa \mathrm{B}$ p65 and cell nuclei were immunostained by Cy3 (red) and DAPI (blue), respectively.

\section{Conflict of Interests}

The authors declare that there is no conflict of interests regarding the publication of this paper.

\section{Acknowledgments}

The samples were kindly provided by professor Zhang from Research Center for Nano-Biomaterials, Analytical \& Testing Center, Sichuan University. The authors acknowledge the National Basic Research Program of China (2012CB933900), Beijing Natural Science Foundation (7144256, 7144257), Doctoral Scientific Fund Project of the Ministry of Education of China (20130001120112), and Beijing Nova program (Z14111000180000).

\section{References}

[1] M. Schuier, D. Trentin, M. Textor, and S. G. Tosatti, "Biomedical interfaces: titanium surface technology for implants and cell carriers," Nanomedicine, vol. 1, no. 4, pp. 449-463, 2006.

[2] D. A. Puleo and A. Nanci, "Understanding and controlling the bone-implant interface," Biomaterials, vol. 20, no. 23-24, pp. 2311-2321, 1999.
[3] S. A. Eming, T. Krieg, and J. M. Davidson, "Inflammation in wound repair: molecular and cellular mechanisms," Journal of Investigative Dermatology, vol. 127, no. 3, pp. 514-525, 2007.

[4] J. M. Schierholz and J. Beuth, "Implant infections: a haven for opportunistic bacteria," Journal of Hospital Infection, vol. 49, no. 2, pp. 87-93, 2001.

[5] Z. Chen, X. Mao, L. Tan et al., "Osteoimmunomodulatory properties of magnesium scaffolds coated with $\beta$-tricalcium phosphate," Biomaterials, vol. 35, no. 30, pp. 8553-8565, 2014.

[6] T. A. Wynn, A. Chawla, and J. W. Pollard, "Macrophage biology in development, homeostasis and disease," Nature, vol. 496, no. 7446, pp. 445-455, 2013.

[7] M. Bartneck, K. H. Heffels, Y. Pan, M. Bovi, G. ZwadloKlarwasser, and J. Groll, "Inducing healing-like human primary macrophage phenotypes by 3D hydrogel coated nanofibres," Biomaterials, vol. 33, no. 16, pp. 4136-4146, 2012.

[8] Y. Zhong and R. V. Bellamkonda, "Dexamethasone-coated neural probes elicit attenuated inflammatory response and neuronal loss compared to uncoated neural probes," Brain Research, vol. 1148, no. 1, pp. 15-27, 2007.

[9] H. Hackstein and A. W. Thomson, "Dendritic cells: emerging pharmacological targets of immunosuppressive drugs," Nature Reviews Immunology, vol. 4, no. 1, pp. 24-34, 2004.

[10] A. Eckhardt, T. Harorli, J. Limtanyakul et al., "Inhibition of cytokine and surface antigen expression in LPS-stimulated 
murine macrophages by triethylene glycol dimethacrylate," Biomaterials, vol. 30, no. 9, pp. 1665-1674, 2009.

[11] D. P. Vasconcelos, A. C. Fonseca, M. Costa et al., "Macrophage polarization following chitosan implantation," Biomaterials, vol. 34, no. 38, pp. 9952-9959, 2013.

[12] D. Naskar, S. Nayak, T. Dey, and S. C. Kundu, "Non-mulberry silk fibroin influence osteogenesis and osteoblast-macrophage cross talk on titanium based surface," Scientific Reports, vol. 4, article 4745, 2014.

[13] A. Kamali, A. Hussain, C. Li et al., "Tribological performance of various $\mathrm{CoCr}$ microstructures in metal-on-metal bearings: the development of a more physiological protocol in vitro," The Journal of Bone and Joint Surgery. British Volume, vol. 92, no. 5, pp. 717-725, 2010.

[14] C.-K. Cheng, N.-K. Yao, H.-C. Liu, and K.-S. Lee, "Influences of configuration changes of the patella on the knee extensor mechanism," Clinical Biomechanics, vol. 11, no. 2, pp. 116-120, 1996.

[15] P. R. Klokkevold, R. D. Nishimura, M. Adachi, and A. Caputo, "Osseointegration enhanced by chemical etching of the titanium surface. A torque removal study in the rabbit," Clinical Oral Implants Research, vol. 8, no. 6, pp. 442-447, 1997.

[16] H. Aita, W. Att, T. Ueno et al., "Ultraviolet light-mediated photofunctionalization of titanium to promote human mesenchymal stem cell migration, attachment, proliferation and differentiation," Acta Biomaterialia, vol. 5, no. 8, pp. 3247-3257, 2009.

[17] Z. Chen, C. Wu, W. Gu, T. Klein, R. Crawford, and Y. Xiao, "Osteogenic differentiation of bone marrow MSCs by $\beta$-tricalcium phosphate stimulating macrophages via BMP2 signalling pathway," Biomaterials, vol. 35, no. 5, pp. 1507-1518, 2014.

[18] K. S. Tan, L. Qian, R. Rosado, P. M. Flood, and L. F. Cooper, "The role of titanium surface topography on J774A.1 macrophage inflammatory cytokines and nitric oxide production," Biomaterials, vol. 27, no. 30, pp. 5170-5177, 2006.

[19] M. A. Baker, S. L. Assis, O. Z. Higa, and I. Costa, "Nanocomposite hydroxyapatite formation on a $\mathrm{Ti}-13 \mathrm{Nb}-13 \mathrm{Zr}$ alloy exposed in a MEM cell culture medium and the effect of $\mathrm{H}_{2} \mathrm{O}_{2}$ addition," Acta Biomaterialia, vol. 5, no. 1, pp. 63-75, 2009.

[20] M. C. Robson, D. A. Dubay, X. Wang, and M. G. Franz, "Effect of cytokine growth factors on the prevention of acute wound failure," Wound Repair and Regeneration, vol. 12, no. 1, pp. 3843, 2004.

[21] S. Franz, S. Rammelt, D. Scharnweber, and J. C. Simon, "Immune responses to implants-a review of the implications for the design of immunomodulatory biomaterials," Biomaterials, vol. 32, no. 28, pp. 6692-6709, 2011.

[22] M. Versaevel, T. Grevesse, and S. Gabriele, "Spatial coordination between cell and nuclear shape within micropatterned endothelial cells," Nature Communications, vol. 3, p. 671, 2012.

[23] R. McBeath, D. M. Pirone, C. M. Nelson, K. Bhadriraju, and C. S. Chen, "Cell shape, cytoskeletal tension, and RhoA regulate stem cell lineage commitment," Developmental Cell, vol. 6, no. 4, pp. 483-495, 2004.

[24] R. Trindade, T. Albrektsson, P. Tengvall, and A. Wennerberg, "Foreign body reaction to biomaterials: on mechanisms for buildup and breakdown of osseointegration," Clinical Implant Dentistry and Related Research, 2014.

[25] T. O. Collier, J. M. Anderson, W. G. Brodbeck, T. Barber, and K. E. Healy, "Inhibition of macrophage development and foreign body giant cell formation by hydrophilic interpenetrating polymer network," Journal of Biomedical Materials Research A, vol. 69, no. 4, pp. 644-650, 2004.

[26] E. Saino, M. L. Focarete, C. Gualandi et al., "Effect of electrospun fiber diameter and alignment on macrophage activation and secretion of proinflammatory cytokines and chemokines," Biomacromolecules, vol. 12, no. 5, pp. 1900-1911, 2011.

[27] W. F. A. den Dunnen, P. H. Robinson, R. van Wessel, A. J. Pennings, M. B. M. van Leeuwen, and J. M. Schakenraad, "Long-term evaluation of degradation and foreignbody reaction of subcutaneously implanted poly(DL-lactide$\varepsilon$-caprolactone)," Journal of Biomedical Materials Research, vol. 36, no. 3, pp. 337-346, 1997.

[28] J. M. Anderson, A. Rodriguez, and D. T. Chang, "Foreign body reaction to biomaterials," Seminars in Immunology, vol. 20, no. 2, pp. 86-100, 2008.

[29] N. A. Athanasou and J. Quinn, "Immunophenotypic differences between osteoclasts and macrophage polykaryons: immunohistological distinction and implications for osteoclast ontogeny and function," Journal of Clinical Pathology, vol. 43, no. 12, pp. 997-1003, 1990.

[30] F. Y. McWhorter, T. Wang, P. Nguyen, T. Chung, and W. F. Liu, "Modulation of macrophage phenotype by cell shape," Proceedings of the National Academy of Sciences of the United States of America, vol. 110, no. 43, pp. 17253-17258, 2013.

[31] T. Albrektsson and C. Johansson, "Osteoinduction, osteoconduction and osseointegration," European Spine Journal, vol. 10, supplement 2, pp. S96-S101, 2001.

[32] P. Palmqvist, E. Persson, H. H. Conaway, and U. H. Lerner, "IL6, leukemia inhibitory factor, and oncostatin $\mathrm{M}$ stimulate bone resorption and regulate the expression of receptor activator of NF-kappaB ligand, osteoprotegerin, and receptor activator of NF-kappaB in mouse calvariae," Journal of Immunology, vol.169, no. 6, pp. 3353-3362, 2002.

[33] A. L. Theiss, J. G. Simmons, C. Jobin, and P. K. Lund, “Tumor necrosis factor (TNF) alpha increases collagen accumulation and proliferation in intestinal myofibroblasts via TNF receptor 2," The Journal of Biological Chemistry, vol. 280, no. 43, pp. 36099-36109, 2005.

[34] Z. Chen, Y. Liu, B. Sun et al., "Polyhydroxylated metallofullerenols stimulate IL- $1 \beta$ secretion of macrophage through TLRs/MyD88/NF- $\kappa$ B pathway and $\mathrm{NLRP}_{3}$ inflammasome activation," Small, vol. 10, no. 12, pp. 2362-2372, 2014.

[35] C. Bogdan, Y. Vodovotz, and C. Nathan, "Macrophage deactivation by interleukin 10," The Journal of Experimental Medicine, vol. 174, no. 6, pp. 1549-1555, 1991.

[36] D. E. Sullivan, M. Ferris, D. Pociask, and A. R. Brody, "Tumor necrosis factor- $\alpha$ induces transforming growth factor$\beta 1$ expression in lung fibroblasts through the extracellular signal-regulated kinase pathway," The American Journal of Respiratory Cell and Molecular Biology, vol. 32, no. 4, pp. 342349, 2005.

[37] S. Lee, J. Choi, S. Shin et al., "Analysis on migration and activation of live macrophages on transparent flat and nanostructured titanium," Acta Biomaterialia, vol. 7, no. 5, pp. 2337-2344, 2011.

[38] M. Tsang, Y. W. Chun, Y. M. Im, D. Khang, and T. J. Webster, "Effects of increasing carbon nanofiber density in polyurethane composites for inhibiting bladder cancer cell functions," Tissue Engineering-Part A, vol. 17, no. 13-14, pp. 1879-1889, 2011. 

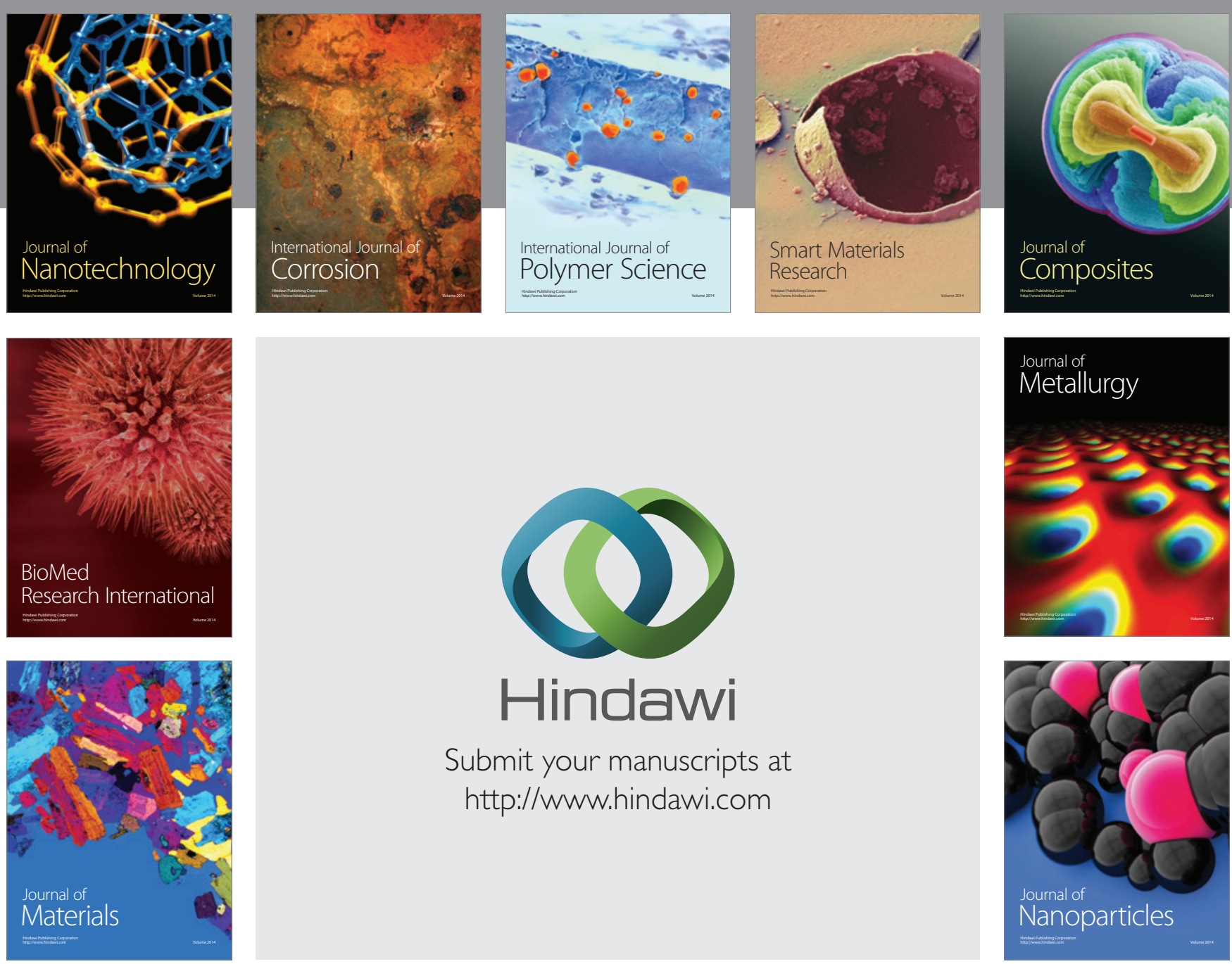

Submit your manuscripts at http://www.hindawi.com
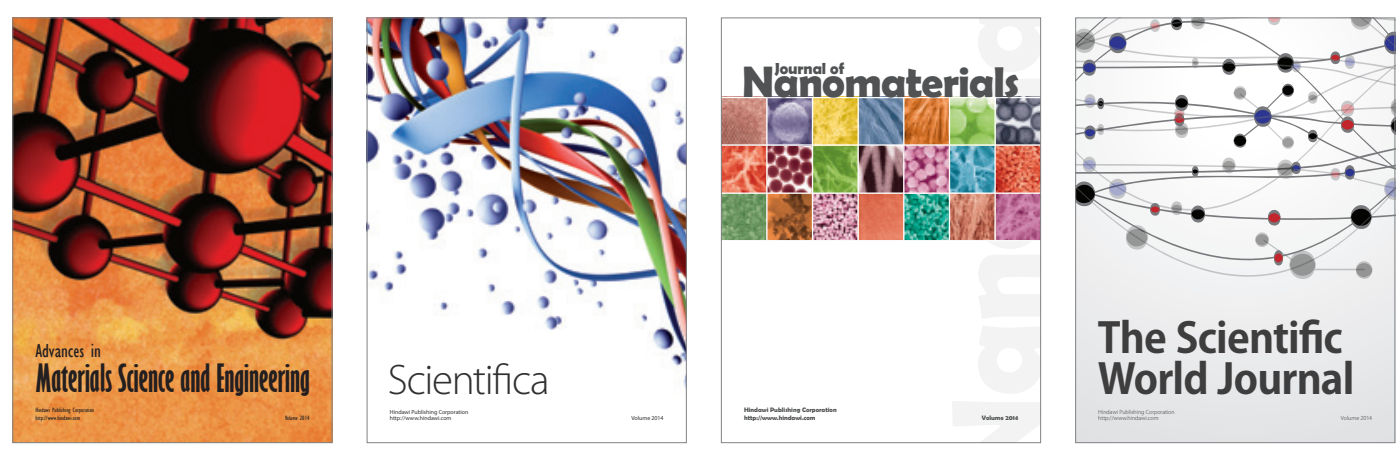

\section{The Scientific World Journal}
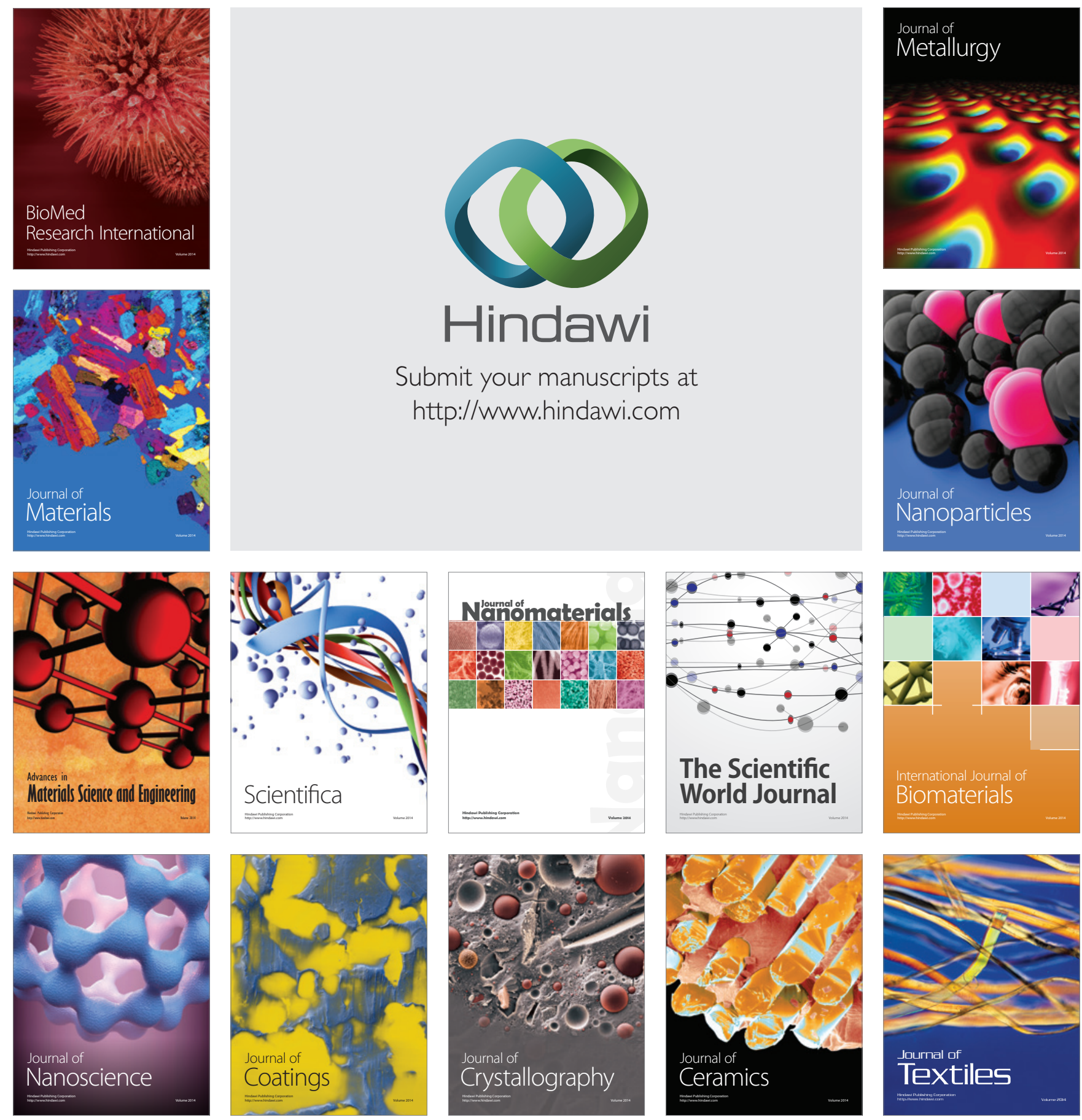